\title{
Ileal Mucosa-Associated Microbiota Overgrowth Associated with Diagnosis and Pathogenesis of Primary Biliary Cholangitis
}

\section{Shogo Kitahata}

Department of Gastroenterology and Metabiology, Ehime University Graduate School of Medicine Yasunori Yamamoto

Endoscopy Center, Ehime University Graduate School of Medicine

\section{Osamu Yoshida}

Department of Gastroenterology and Metabiology, Ehime University Graduate School of Medicine

\section{Yoshio Tokumoto}

Department of Community Medicine, Ehime University Graduate School of Medicine

\section{Tomoe Kawamura}

Endoscopy Center, Ehime University Graduate School of Medicine

\section{Teru Kumagi}

Ehime University Hospital Postgraduate Medical Education Center

\section{Masashi Hirooka}

Department of Gastroenterology and Metabiology, Ehime University Graduate School of Medicine

\section{Eiji Takeshita}

Department of Inflammatory Bowel Diseases and Therapeutics, Ehime University Graduate School of Medicine

\section{Masanori Abe}

Department of Gastroenterology and Metabiology, Ehime University Graduate School of Medicine

\section{Yoshiou Ikeda}

Endoscopy Center, Ehime University Graduate School of Medicine

Yoichi Hiasa ( $\nabla$ hiasa@m.ehime-u.ac.jp )

Department of Gastroenterology and Metabiology, Ehime University Graduate School of Medicine

\section{Research Article}

Keywords: pathogenesis of primary biliary cholangitis (PBC), mucosa-associated microbiota, ribosomal RNA, Sphingomonadaceae, Pseudomonas

Posted Date: June 28th, 2021

DOI: https://doi.org/10.21203/rs.3.rs-645870/v1 
License: (c) (i) This work is licensed under a Creative Commons Attribution 4.0 International License. Read Full License 


\section{Abstract \\ Background}

The gut microbiota has potential implications in the pathogenesis of primary biliary cholangitis (PBC). However, little is known about the significance of small intestinal mucosa-associated microbiota (MAM) in PBC. We aimed to investigate the ileal MAM profile and identify its association with liver pathology in patients with PBC.

\section{Methods}

Forty-three patients with PBC and 24 healthy controls who underwent colonoscopy at our hospital between March 2018 and January 2020 were enrolled in a cross-sectional study. We performed 16S ribosomal RNA gene sequencing of MAM samples obtained from the mucosa of the terminal ileum of all subjects. We also examined the relationship between the abundance of ileal MAM and chronic nonsuppurative destructive cholangitis using liver specimens from patients with PBC.

\section{Results}

Dysbiosis of ileal MAM was observed in patients with PBC, with a characteristic overgrowth of Sphingomonadaceae and Pseudomonas. Multivariate analysis showed that the overgrowth of Sphingomonadaceae and Pseudomonas is an independent association factor for PBC. Moreover, the abundance of Sphingomonadaceae was associated with chronic nonsuppurative destructive cholangitis in PBC.

\section{Conclusions}

Overgrowth of Sphingomonadaceae and Pseudomonas in ileal MAM is an independent association factor for diagnosing PBC. Sphingomonadaceae may be particularly associated with the pathological development of PBC.

\section{Introduction}

Primary biliary cholangitis (PBC) is a chronic liver disease resulting from progressive, immune-mediated destruction of the small interlobular bile ducts. It leads to progressive intrahepatic cholestasis and eventually fibrosis and cirrhosis of the liver $[1,2]$. The etiology of PBC is attributed to a combination of genetic predispositions and environmental triggers such as the gut microbiota [3]. A characteristic of this disease is the presence of antimitochondrial antibodies (AMA), which are autoantibodies against mitochondrial pyruvate dehydrogenase complex E2 (PDC-E2) [4,5]. Previous studies have reported that Novosphingobium aromaticivorans, a bacterium belonging to the family of Sphingomonadaceae, 
produced a homologous enzyme against PDC-E2 [6-8]. Moreover, mice infected with $N$. aromaticivorans show antibodies against microbial PDC-E2 and its mitochondrial counterpart, as well as chronic T cellmediated autoimmunity against small bile ducts [9]. Fecal microbiota profiles of patients with PBC have a significant reduction in microbial diversity compared with healthy controls (HCs), suggesting gut microbiota as a potential therapeutic target and diagnostic biomarker for PBC [10, 11]. However, overgrowth of any particular bacterium, including Sphingomonadaceae, has not been identified in the fecal microbiome of patients with PBC. Therefore, the infection site of the specific bacteria involved in the pathogenesis and progression of $\mathrm{PBC}$ remains unknown.

The gut microbiota comprises luminal microbiota (LM), fecal microbiota, and mucosa-associated microbiota (MAM) present in the intestinal mucosa. MAM affects epithelial and mucosal functions more remarkably than LM, and hence, it is potentially more involved in PBC pathophysiology than LM [12]. However, the MAM profile in patients with PBC is unknown. The ileal MAM could be associated with the synthesis of bile acids that are involved in PBC pathogenesis $[13,14]$. Most bile acids return to the liver via the enterohepatic circulation at the terminal ileum [15]. Thus, in cholestatic disease, the profile of ileal MAM is an important factor in the pathophysiology of this disease. Recently, a brushing method was proposed to evaluate MAM [16]. In the present study, we aimed to investigate the ileal MAM profile and identify its association with liver pathology in patients with PBC.

\section{Results}

\section{Clinical characteristics of the subjects}

The clinical characteristics of patients with PBC and HCs are summarized in Table 1. Although the number of women in the PBC group was significantly greater than that in the HC group, their mean age and body mass indices were comparable. The levels of $\mathrm{y}$-glutamyl transpeptidase, ALP, total bile acid, and immunoglobulin M were higher in patients with PBC than in those of the HCs. High levels of total bilirubin in the sera of HCs were observed, although within the standard range.

\section{Dysbiosis in the MAM of patients with PBC}

An unweighted, UniFrac-based, principal coordinate analysis showed relatively weak clustering of MAM between patients with PBC and HCs (PERMANOVA, pseudo-F: 1.44, $P=0.054$, Figure 1a).

Gut microbiota richness, determined by the Chao- 1 index, was lower in patients with PBC than in $\mathrm{HCs}(P=$ 0.039, Wilcoxon rank-sum test; Figure 1b). However, the Shannon index, which measures both richness and evenness, was not significantly different among the two groups $(P>0.05$, Figure $1 \mathrm{c})$.

\section{Characterization of MAM present in patients with PBC and HCs}

To estimate microbiota diversity in PBC patient samples, we assessed the relative abundance of taxa in both groups. Figure 2a shows the relative bacterial abundance at the phylum level in the samples from both groups. The most dominant phylum was Firmicutes with an average relative abundance of $40.5 \%$ 
and $40.3 \%$ in the PBC and $\mathrm{HC}$ groups, respectively. However, no significant differences were observed at the phylum level between the groups. Phylum level analysis showed that the relative abundance of TM7 was significantly lower in the PBC group than in the $\mathrm{HC}$ group $(P=0.00725$, Figure $2 \mathrm{~b})$.

At the class level, Alphaproteobacteria were more abundant in the PBC group than in the HC group, whereas TM7_3 was significantly decreased in the PBC group (Figure 3a). At the order level, Sphingomonadales and Rhizobiales, of the class Alphaproteobacteria and Pseudomonadales, respectively, were more abundant in the PBC group than in the HC group. CWO4O and a species belonging to an unknown order that belongs to the TM7_3 class were considerably decreased in the PBC group (Figure 3b). At the family level, four bacterial taxa including Sphingomonadaceae, Pseudomonadaceae, Methylobacteriaceae, and Moraxellaceae were more abundant in the PBC group than in the HC group, whereas Leptotrichiaceae, Burkholderiaceae, Comamonadaceae, F16, and an unknown family belonging to the class TM7_3 were considerably decreased in the PBC group (Figure 3c). The abundance of 18 bacterial genera differed between the groups $(P<0.05)$ (Figure 3d). Eleven genera (Leptotrichia, Morganella, Lautropia, Mogibacterium, Atopobium, Bulleidia, Eikenella, Paludibacter, an unknown genus belonging to the family Streptococcaceae (Streptococcaceae,g), an unknown genus belonging to the class $T M 7 \_3$, and $F 16, g$ (an unknown genus of the family $F 16$ ) were substantially abundant in HCs (Figure 3d, red bar). Conversely, seven genera

(Sphingomonas, Pseudomonas, Methylobacterium, Carnobacterium, Acinetobacter, Curvibacter, and the unknown genus Clostridiaceae belonging to the family Clostridiaceae) were substantially abundant in the PBC group (Figure 3d, green bar). Notably, Sphingomonas and Pseudomonas were associated with PBC from the order to the genus levels. Of these, we further focused on Sphingomonadaceae and Pseudomonas, which have been previously reported to be related to PBC [9].

\section{Relationship between specific bacteria and treatment-naïve PBC}

To rule out the effects of therapeutic agents, we evaluated the relative abundance of

Sphingomonadaceae and Pseudomonas in treatment-naïve patients with PBC or patients receiving UDCA, the main treatment for PBC. No significant difference was observed when the abundance of Sphingomonadaceae and Pseudomonas was compared between the UDCA-naïve PBC $(\mathrm{n}=6)$ and UDCAtreated $(n=28)$ groups (Supplementary figure 1$)$.

\section{Analysis of association factors contributing to PBC using microbiome signature}

To explore whether the gut microbiome can help determine the PBC status, we determined the abundance of Sphingomonadaceae and Pseudomonas in the PBC groups by receiver operating characteristic analysis. The AUC values obtained were 0.745 ( $95 \% \mathrm{Cl} 0.63$ to 0.87 , Supplementary figure 2a) and 0.735 (95\% $\mathrm{Cl} 0.60$ to 0.87 , Supplementary figure $2 \mathrm{~b})$, respectively. The cut-off values were 0.0000179 and 0.0000684 for Sphingomonadaceae and Pseudomonas, respectively. Based on this threshold, patients with PBC were divided into four subgroups, i.e., Sphingomonadaceae rich and deficient, and Pseudomonas rich and deficient. Since PBC frequently occurs in middle-aged women, univariate analysis of these bacteria was performed considering age and sex as the evaluation 
parameters. Factors (Sphingomonadaceae rich, Pseudomonas rich, and sex) identified using univariate analysis were subject to multivariate analysis. Among Sphingomonadaceae, Pseudomonas, and sex, which were significant in univariate analysis, all items remained significant after multivariate analysis $(P$ $=0.0196, P=0.0438, P=0.0337$, Table 2).

\section{Relationship between specific bacteria and chronic non-suppurative destructive cholangitis (CNSDC)}

We evaluated the relative abundance of Sphingomonadaceae and Pseudomonas in 27 patients who had undergone liver biopsy to investigate whether these bacteria are associated with the pathology of PBC. A comparison of the abundance of Sphingomonadaceae in CNSDC-positive PBC $(n=22)$ and CNSDCnegative PBC $(n=5)$ groups showed that Sphingomonadaceae were more abundant in the CNSDCpositive group than in the CNSDC-negative group $(P=0.0194$, Figure 4a). Similar results were obtained with the Sphingomonadaceaerich group based on the cut-off value obtained by ROC $(P=0.00981$, Table $3)$. On the other hand, there was no difference in the abundance of Pseudomonas between the CNSDCpositive group and CNSDC-negative group (Figure 4b).

\section{Discussion}

This study was designed to evaluate the ileal MAM profile and identify its association with liver pathology in patients with PBC. We found dysbiosis in the ileal MAM of patients with PBC. Furthermore, the abundance of Sphingomonadaceae and Pseudomonas in the MAM of patients with PBC was significantly increased compared with that in $\mathrm{HCs}$ and showed an independent association for PBC diagnosis. In particular, the amount of Sphingomonadaceae was associated with the CNSDC in the liver biopsy samples of patients with PBC. Previous studies have reported a significant reduction in intraindividual microbial diversity in the fecal samples of patients with PBC [10,11]. This finding was consistent with the results of the present study. However, overgrowth of any particular bacteria involved in the pathogenesis and progression of PBC has not been identified in the fecal microbiome of patients with PBC. The present study is the first report to identify an overgrowth of Sphingomonadaceae and Pseudomonas, which activates autoimmunity and cross-reacts with the PBC antigen in the ileal MAM of patients with PBC.

The bacteria that belong to the division/phylum Proteobacteria and class Alphaproteobacteria have been remarkably associated with autoimmunity [17]. The cell wall of Sphingomonadaceae spp. and those belonging to the Alphaproteobacteria class is unusual as it contains glycosphingolipids (GSLs) instead of lipopolysaccharides $[18,19]$. The unique cell wall of these bacteria activates distinct innate immune pathways that may trigger the development of liver-specific pathological conditions [17]. Mice infected with $N$. aromaticivorans, belonging to the genus Sphingomonadaceae, developed antibodies against PDC-E2 and had liver histology similar to patients with PBC [9]. The model was postulated because natural killer T (NKT) cells specifically recognized the GSLs of various bacterial strains belonging to the genus Sphingomonadaceae through their semi-invariant CD1d-restricted T-cell receptor [9]. Sphingomonadaceae GSLs, which have a similar structure to a-GalCer, bind to mouse CD1d and 
stimulate Va14iNKT cells independent of antigen-presenting cell activation and interleukin-12 [20, 21]. NKT cells accumulate in the liver [22], and the proportion of NKT cells in the liver has been reported to be significantly higher in patients with PBC than in healthy individuals [23]. Additionally, CD1d is focally expressed on small bile duct epithelial cells in patients with PBC but not in healthy individuals [24]. Previous studies performed using fecal samples of patients with PBC reported no significant differences in the abundance of Sphingomonadaceae [10]. The MAM of the small intestine is different from the LM and is considered to be more deeply involved in the pathophysiology of PBC [16]. Our study showed that patients with $\mathrm{PBC}$ have significantly more Sphingomonadaceae than HCs, with Sphingomonadaceae present in patients with PBC with or without UDCA treatment. Additionally, the dominance of Sphingomonadaceae detected with receiver operating characteristic curve and multivariate analysis suggested Sphingomonadaceae as one of the factors involved in PBC. Our findings point towards the terminal ileum as the site of infection.

Pseudomonas primarily causes urinary tract infection, acts as an environmental risk factor for PBC, and causes the production of PBC autoantibodies [25-27]. Previous studies on patients with PBC showed extensive cross-reactivity between the dominant B- and T-cell epitopes of human PDC-E2 and microbial mimics, suggesting the role of microbial infection in the induction of antimitochondrial antibodies through the mechanism of molecular mimicry [28]. Pseudomonas aeruginosa, $N$. aromaticivorans, and Escherichia coli contain proteins that are homologous with PDC-E2 [8, 9]. Pseudomonas species crossreacting with PDC-E2-reactive $\mathrm{CD} 4^{+}$and $\mathrm{CD} 8^{+} \mathrm{T}$ cells have been identified and show potential agonistic effects $[25,26]$. A previous study identified Pseudomonas species in the liver tissue of patients with PBC [29]. Pseudomonas may therefore have a significant effect on immunological tolerance and autoimmunity in patients with PBC. The dominance of Pseudomonas analyzed through receiver operating characteristic curve and multivariate analysis suggested that Pseudomonas is one of the factors involved in PBC etiology similar to Sphingomonadaceae.

CNSDC, one of the diagnostic criteria for PBC, is a typical early hepatopathological finding of PBC. Previous studies have reported the production of bacterially induced PBC-like CNSDC in mice [30], but there are no reports of an association between CNSDC and specific bacteria in humans. In this study, we identified a relationship between CNSDC and Sphingomonadaceae, which stimulates NKT cells. Bile duct epithelial staining of CD1d is a common feature in early-stage PBC but is rarely present in late-stage PBC [24]. Innate immunity triggered by Sphingomonadaceae may be a contributing factor for the pathophysiology of early PBC.

Our study had several limitations. First, we were unable to identify the variability between patients at different stages because we included a small number of patients who were in the advanced stage of PBC. Therefore, it is necessary to analyze independent cohorts comprising early- and late-stage patients with PBC. Second, this was a cross-sectional study, and therefore, we cannot conclude that there is a causal relationship between MAM and PBC. Evaluation of the effect of treatment against Sphingomonadaceae and Pseudomonas to inhibit the development of PBC should be assessed in vivo. Third, the time of liver biopsy was different from that of bacterial collection. Although evaluation at the 
same time is optimal, the different time of collection has a small effect because the gut microbiota does not change unless there are antibiotics present or major events have occurred. The main strength of this study is that it is the first to identify the overgrowth of specific bacteria in ileal MAM of PBC, and these bacteria are potentially associated in the pathogenesis of PBC.

In conclusion, we revealed dysbiosis in the MAM profile of the small intestine in patients with PBC and characteristic bacterial overgrowth that has been reported to be associated with PBC.

Sphingomonadaceae may be associated with the pathological development of PBC. Our results contribute to the development of effective antibiotic treatment strategies for PBC.

\section{Methods}

\section{Subjects}

In this study, 41 patients with PBC and $24 \mathrm{HCs}$ underwent colonoscopy at the Ehime University Hospital (Ehime, Japan) between March 2018 and January 2020. The selection criteria for the subjects were as follows: [a] Biochemical evidence of cholestasis (increase in the serum alkaline phosphatase [ALP] level), [b] AMA positivity, and [c] histological evidence of the destruction of interlobular bile ducts and nonsuppurative destructive cholangitis per liver biopsy. Patients who fulfilled at least two of the three criteria were included in this study [31]. The exclusion criteria were as follows: viral hepatitis, autoimmune hepatitis, biliary tract system and pancreatic disease, past surgical history of gastroenterological diseases, and past history of liver transplantation. The 16S rRNA sequence analysis was performed on 34 patients with PBC and $21 \mathrm{HCs}$, who had not received antibiotics within 2 months of sample collection and did not have gastrointestinal cancer (Supplementary figure 3). The groups were further subdivided based on whether patients received ursodeoxycholic acid (UDCA) treatment. In addition, we analyzed a subgroup of patients who had undergone liver biopsy. All the subjects provided written informed consent and the study protocol was developed in accordance with the ethical guidelines of the 1964 Declaration of Helsinki and later versions. All procedures followed were in accordance with the ethical standards of the responsible committee on human experimentation (institutional and national). This study was approved by the ethics committee of Ehime University of Medical Science (approval no. 1610012). All the patients were managed at the Division of Gastroenterology of Ehime University Hospital. The study was registered at the University Hospital Medical Information Network Center (UMIN 000040177). All authors had access to the study data, and reviewed and approved the final manuscript.

\section{Sample collection and DNA extraction for MAM study}

Samples from the terminal ileum were collected by gently brushing the mucosal surfaces using RX cytology brushes (Boston Scientific, Marlborough, MA, USA) according to a previously reported technique [16] and frozen at $-80^{\circ} \mathrm{C}$. DNA extraction was performed using the QIAamp UCP Pathogen Mini Kit (Qiagen, Hilden, Germany). 
Using the MiSeq system (Illumina, San Diego, CA, USA), 16S rRNA gene sequencing was performed as described previously [32]. The V3-V4 region of the bacterial 16S ribosomal RNA gene was amplified using the KAPA HiFi HotStart PCR kit (Kapa Biosystems, Woburn, MA, USA) and barcode-indexed primers 341F (5'-CCTACGGGNGGCWGCAG-3') and 806R (5'-GACTACHVGGGTATCTAATCC-3'). The amplicons were purified using AMpureXP (Beckman Coulter, Tokyo, Japan) and quantified using Qubit (Thermo Fisher Scientific, Waltham, MA, USA). The purified amplicons were pooled in equimolar concentrations $(1.20 \mathrm{ng} / \mu \mathrm{L})$ and sequenced.

\section{Data analysis}

The 16S rRNA sequence analysis was performed using the QIIME suite of software tools (v1.9.1) [33]. Operational taxonomic units were picked from filtered sequence reads (Phred score $\geq$ Q33) by a closedreference operational taxonomic unit-picking method on the basis of $97 \%$ identity with the Greengenes database (v13.5) [34]. Chimeric sequences were trimmed using the ChimeraSlayer method [35]. Chao-1 diversity indices were used to compare the diversity of gut microbiota profiles between patients with PBC and HCs. For comparing beta diversity, weighted and unweighted UniFrac distances were calculated [36]. To determine the significance of the inter- and intra-group distance differences, non-parametric $t$-tests were performed with 999 permutations. For multivariate analysis of variance, permutational analysis of variance (PERMANOVA) was used with 999 permutations.

\section{Statistical analysis}

Statistical analysis was performed using the paired Wilcoxon rank-sum test and a P-value $<0.05$ was considered statistically significant. All statistical analyses were performed using R package (V.3.5.0). To evaluate the discriminatory ability of the gut microbiota of patients with PBC, operating characteristic curves (receiving operational curve [ROC]) were constructed and the area under the curve (AUC) was calculated. For correlation analysis, Spearman's rank test was performed. Logistic regression tests were used to regress the relative abundance of bacteria against gender and age. Differences in the ratios of pathological findings according to the bacteria associated with PBC were examined by the $\chi 2$ test.

\section{Declarations}

\section{Data availability}

The data that support the findings of this study are available from the corresponding author, $\mathrm{YH}$, upon reasonable request.

\section{Acknowledgements}

We thank Takana Fujino, Chie Takeichi, Kenji Tanimoto, and Ayumi Sumizaki for their technical assistance.

\section{Author contributions}


Study concept and design: YY.

Generation, collection, assembly, analysis, and/or interpretation of data: SK, YY, OY, YT, TK, TK, MH, ET, MA, YI, YH.

Drafting or revision of manuscript: $\mathrm{YY}, \mathrm{YH}$.

Competing interests: The authors declare no competing interests. The authors received no financial support for the research, authorship, and/or publication of this article.

\section{References}

1. Lindor, K. D. et al. Primary biliary cirrhosis. Hepatology, 50, 291-308 (2009).

2. Kumagi, T. \& Heathcote, E. J. Primary biliary cirrhosis. Orphanet. J. Rare Dis, 3, 1 (2008).

3. Selmi, C. et al. Primary biliary cirrhosis in monozygotic and dizygotic twins: genetics, epigenetics, and environment. Gastroenterology, 127, 485-492 (2004).

4. Oertelt, S. et al. A sensitive bead assay for antimitochondrial antibodies: Chipping away at AMAnegative primary biliary cirrhosis. Hepatology, 45, 659-665 (2007).

5. Moteki, S. et al. Epitope mapping and reactivity of autoantibodies to the E2 component of 2oxoglutarate dehydrogenase complex in primary biliary cirrhosis using recombinant 2-oxoglutarate dehydrogenase complex. Hepatology, 23, 436-444 (1996).

6. Padgett, K. A. et al. Phylogenetic and immunological definition of four lipoylated proteins from Novosphingobium aromaticivorans, implications for primary biliary cirrhosis. J. Autoimmun, 24, 209-219 (2005).

7. Kaplan, M. M. Novosphingobium aromaticivorans: a potential initiator of primary biliary cirrhosis. Am. J. Gastroenterol, 99, 2147-2149 (2004).

8. Selmi, C. et al. Patients with primary biliary cirrhosis react against a ubiquitous xenobioticmetabolizing bacterium. Hepatology, 38, 1250-1257 (2003).

9. Mattner, J. et al. Liver autoimmunity triggered by microbial activation of natural killer T cells. Cell Host Microbe, 3, 304-315 (2008).

10. Tang, R. et al. Gut microbial profile is altered in primary biliary cholangitis and partially restored after UDCA therapy. Gut, 67, 534-541 (2018).

11. Furukawa, M. et al. Gut dysbiosis associated with clinical prognosis of patients with primary biliary cholangitis. Hepatol. Res, 50, 840-852 (2020).

12. Ringel, Y. et al. High throughput sequencing reveals distinct microbial populations within the mucosal and luminal niches in healthy individuals. Gut Microbes, 6, 173-181 (2015).

13. Li, Y., Tang, R., Leung, P. S. C., Gerschwin, M. E. \& Ma, X. Bile acids and intestinal microbiota in autoimmune cholestatic liver diseases. Autoimmun. Rev, 16, 885-896 (2017). 
14. Chen, W. et al. Comprehensive analysis of serum and fecal bile acid profiles and interaction with gut microbiota in primary biliary cholangitis. Clin. Rev. Allergy Immunol, 58, 25-38 (2020).

15. Hamer, H. M., De Preter, V., Windey, K. \& Verbeke, K. Functional analysis of colonic bacterial metabolism: relevant to health? Am. J. Physiol. Gastrointest. Liver Physiol, 302, G1-G9 (2012).

16. Nishino, K. et al. Analysis of endoscopic brush samples identified mucosa-associated dysbiosis in inflammatory bowel disease. J. Gastroenterol, 53, 95-106 (2018).

17. Mohammed, J. P. \& Mattner, J. Autoimmune disease triggered by infection with alphaproteobacteria. Expert Rev. Clin. Immunol, 5, 369-379 (2009).

18. Kawahara, K., Kuraishi, H. \& Zähringer, U. Chemical structure and function of glycosphingolipids of Sphingomonas spp and their distribution among members of the alpha-4 subclass of Proteobacteria. J. Ind. Microbiol. Biotechnol, 23, 408-413 (1999).

19. Kawahara, K., Moll, H., Knirel, Y. A. \& Zähringer, U. Structural analysis of two glycosphingolipids from the lipopolysaccharide-lacking bacterium Sphingomonas capsulata. Eur. J. Biochem, 267, 18371846 (2000).

20. Kinjo, Y. et al. Recognition of bacterial glycosphingolipids by natural killer T cells. Nature, 434, 520525 (2005).

21. Mattner, J. et al. Exogenous and endogenous glycolipid antigens activate NKT cells during microbial infections. Nature, 434, 525-529 (2005).

22. Geissmann, F. et al. Intravascular immune surveillance by CXCR6 + NKT cells patrolling liver sinusoids. PLoS Biol, 3, e113 (2005).

23. Kita, H. et al. Quantitation and phenotypic analysis of natural killer T cells in primary biliary cirrhosis using a human CD1d tetramer. Gastroenterology, 123, 1031-1043 (2002).

24. Tsuneyama, K. et al. Increased CD1d expression on small bile duct epithelium and epithelioid granuloma in livers in primary biliary cirrhosis. Hepatology, 28, 620-623 (1998).

25. Kita, H. et al. Analysis of TCR antagonism and molecular mimicry of an HLA-A0201-restricted CTL epitope in primary biliary cirrhosis. Hepatology, 36, 918-926 (2002).

26. Shimoda, S. et al. Mimicry peptides of human PDC-E2 163-176 peptide, the immunodominant T-cell epitope of primary biliary cirrhosis. Hepatology, 31, 1212-1216 (2000).

27. Kumagi, T., Abe, M., Ikeda, Y. \& Hiasa, Y. Infection as a risk factor in the pathogenesis of primary biliary cirrhosis: pros and cons. Dis. Markers, 29, 313-321 (2010).

28. Bogdanos, D. P. et al. Microbial mimics are major targets of crossreactivity with human pyruvate dehydrogenase in primary biliary cirrhosis. J. Hepatol, 40, 31-39 (2004).

29. Harada, K., Tsuneyama, K., Sudo, Y., Masuda, S. \& Nakanuma, Y. Molecular identification of bacterial $16 \mathrm{~S}$ ribosomal RNA gene in liver tissue of primary biliary cirrhosis: is Propionibacterium acnes involved in granuloma formation? Hepatology, 33, 530-536 (2001).

30. Haruta, I. et al. Involvement of commensal bacteria may lead to dysregulated inflammatory and autoimmune responses in a mouse model for chronic nonsuppurative destructive cholangitis. J. Clin. 
Immunol, 32, 1026-1037 (2012).

31. Chazouillères, O. et al. Primary biliary cirrhosis-autoimmune hepatitis overlap syndrome: clinical features and response to therapy. Hepatology, 28, 296-301 (1998).

32. Caporaso, J. G. et al. Ultra-high-throughput microbial community analysis on the Illumina HiSeq and MiSeq platforms. ISME J, 6, 1621-1624 (2012).

33. Caporaso, J. G. et al. QIIME allows analysis of high-throughput community sequencing data. Nat. Methods, 7, 335-336 (2010).

34. Edgar, R. C. Search and clustering orders of magnitude faster than BLAST. Bioinformatics, 26, 24602461 (2010).

35. Haas, B. J. et al. Chimeric $16 \mathrm{~S}$ rRNA sequence formation and detection in Sanger and 454pyrosequenced PCR amplicons. Genome Res, 21, 494-504 (2011).

36. Lozupone, C. \& Knight, R. UniFrac: a new phylogenetic method for comparing microbial communities. Appl. Environ. Microbiol, 71, 8228-8235 (2005).

\section{Tables}

Table 1. Baseline characteristics of 34 patients with $\mathrm{PBC}^{\mathrm{a}}$ and 21 healthy controls 


\begin{tabular}{|c|c|c|c|}
\hline & $\begin{array}{l}\mathrm{PBC}^{\mathrm{a}} \\
(n=34)\end{array}$ & $\begin{array}{l}\text { Healthy control } \\
(n=21)\end{array}$ & $P$ value** \\
\hline Age, years * & $66(58-72)$ & $69(55-73)$ & 0.795 \\
\hline Gender, male/female & $2 / 32$ & $9 / 12$ & 0.001 \\
\hline $\mathrm{BMI}^{\mathrm{a}}, \mathrm{kg} / \mathrm{m}^{2}$ * & $22.4(20.1-24.9)$ & $22.8(20.8-24.9)$ & 0.822 \\
\hline$A L T^{a}, U / L$ * & $18(14-22.8)$ & $17(15-22)$ & 0.815 \\
\hline $\mathrm{AST}^{\mathrm{a}}, \mathrm{U} / \mathrm{L}$ * & $26.5(22-30.8)$ & $22(19-26)$ & 0.081 \\
\hline $\mathrm{GGT}^{\mathrm{a}}, \mathrm{U} / \mathrm{L}$ * & $34.5(21-63.8)$ & $19(17-35)$ & 0.01 \\
\hline$A L P^{a}, U / L$ * & $275(223-328)$ & $225(181-245)$ & 0.003 \\
\hline $\mathrm{T}-\mathrm{Bil}^{\mathrm{a}}, \mathrm{mg} / \mathrm{dL}$ * & $0.6(0.4-0.8)$ & $0.8(0.7-1.0)$ & 0.016 \\
\hline $\mathrm{PT}^{\mathrm{a}}, \%$ * & $105(95-118)$ & $103(94.8-113)$ & 0.457 \\
\hline Total bile acid, $\mu \mathrm{mol} / \mathrm{L}$ * & $11.9(8-18.7)$ & $4.1(2.8-6.9)$ & 0.0001 \\
\hline $\operatorname{lgM}{ }^{a}, \mathrm{mg} / \mathrm{dL}$ * & $137.5(96.7-192)$ & $103(52-141.5)$ & 0.044 \\
\hline $\lg \mathrm{G}^{\mathrm{a}}, \mathrm{mg} / \mathrm{dL}$ * & $1315(1162-1526)$ & $1350(1245-1470)$ & 0.67 \\
\hline $\mathrm{AMA}^{\mathrm{a}}, \mathrm{n}(+\%)$ & $26 / 8(76.5 \%)$ & & \\
\hline $\mathrm{UDCA}^{\mathrm{a}}, \mathrm{n}(+\%)$ & $28 / 6(82.4 \%)$ & & \\
\hline Clinical cirrhosis, n (+\%) & 0/33 (0\%) & & \\
\hline
\end{tabular}

*Median (interquartile range)

${ }^{\star *}$ Comparisons using Wilcoxon rank-sum test and Fisher's exact test, $P<0.05$ defined as threshold of significance.

aAbbreviations: PBC, primary biliary cholangitis; $\mathrm{BMI}$, body mass index; ALT, alanine aminotransferase; AST, aspartate aminotransferase; GGT, y-glutamyltranspeptidase; ALP, alkaline phosphatase; T-Bil, total bilirubin; PT, prothrombin time; IgM, immunoglobulin M; IgG, immunoglobulin G; AMA, antimitochondria antibody; UDCA, ursodeoxycholic acid.

Table 2. Multivariate analysis for ability to diagnose $\mathrm{PBC}^{\mathrm{a}}$ 


\begin{tabular}{|lllll|}
\hline & Univariate & & Multivariate & \\
\hline & OR $\left(95 \% \mathrm{Cl}^{\mathrm{a}}\right)$ & Pvalue* & OR $\left(95 \% \mathrm{Cl}^{\mathrm{a}}\right)$ & Pvalue* $^{\star}$ \\
\hline Age, years & $0.99(0.94-1.05)$ & 0.96 & & \\
\hline Female & $12(2.63-63.7)$ & 0.00353 & $8.64(1.18-63.3)$ & 0.0337 \\
\hline $\begin{array}{l}\text { Sphingomonadaceae } \\
\text { rich** }\end{array}$ & $9.69(2.38-39.5)$ & 0.00153 & $6.23(1.34-29)$ & 0.0196 \\
\hline $\begin{array}{l}\text { Pseudomonas } \\
\text { rich }\end{array}$ & $7.58(2.18-26.4)$ & 0.00144 & $4.49(1.04-19.3)$ & 0.0438 \\
\hline
\end{tabular}

*Comparisons using Wilcoxon rank-sum test and Fisher's exact test, $P<0.05$ defined as threshold of significance.

**The cutoff values were 0.0000179 .

$\star \star \star T h e ~ c u t o f f$ values were 0.0000684

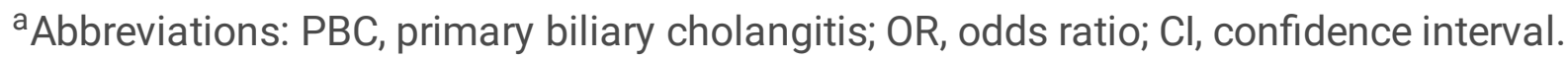

Table 3. Relationship between the mucosa-associated microbiota and CNSDC ${ }^{a}$

\begin{tabular}{|c|c|c|c|}
\hline & $\begin{array}{l}\text { CNSDC }^{\text {a }} \text { positive } \\
(n=22)\end{array}$ & $\begin{array}{l}\text { CNSDC }^{\text {a }} \text { negative } \\
(n=5)\end{array}$ & $P$ value* \\
\hline $\begin{array}{l}\text { Sphingomonadaceae } \\
\text { Rich / Deficient** }\end{array}$ & $15 / 7$ & $0 / 5$ & 0.00981 \\
\hline 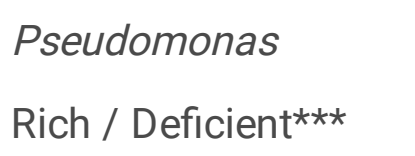 & $18 / 4$ & $4 / 1$ & 1 \\
\hline
\end{tabular}

*Comparisons using Wilcoxon rank-sum test and Fisher's exact test, $P<0.05$ defined as threshold of significance.

$\star \star$ The cutoff values were 0.0000179 .

$\star \star \star T h e ~ c u t o f f$ values were 0.0000684

aAbbreviations: CNSDC, chronic non-suppurative destructive cholangitis.

\section{Figures}




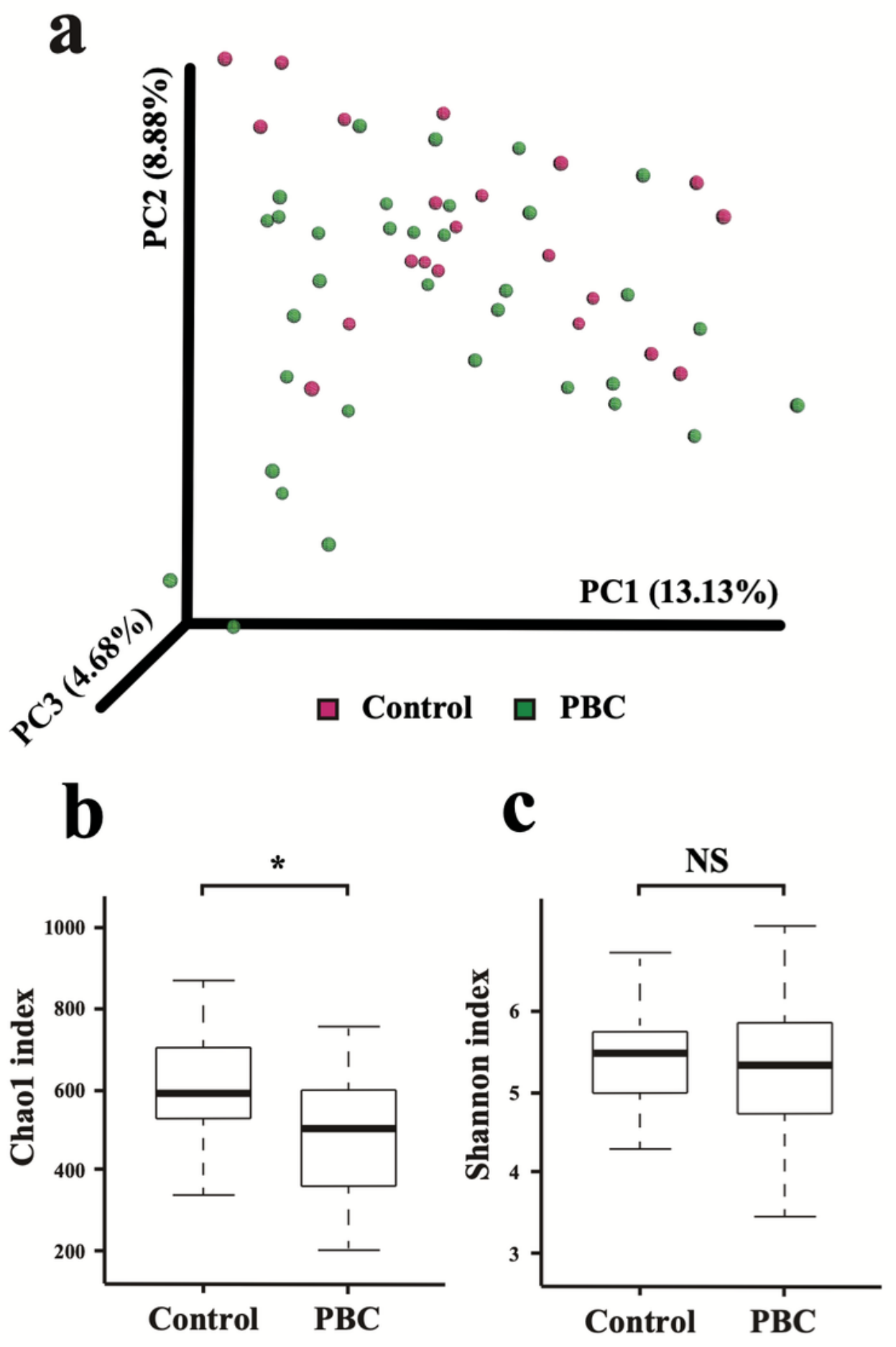

\section{Figure 1}

Comparison of MAM diversity between patients with PBC and HCs (a) Principal coordinate analysis of unweighted UniFrac analysis showed a somewhat weak clustering of the MAM between the patients with PBC and HCs (pseudo-F: 1.44, P = 0.054, PERMANOVA). (b) Changes in the mucosa-associated microbiota diversities in patients with PBC $(n=34)$ compared with HCs $(n=21)$. An a-diversity, illustrated by microbial richness (Chao-1 index), was reduced in the PBC group $(P=0.039$, Wilcoxon rank-sum test). 
${ }^{*} \mathrm{P}<$ 0.05. (c) No difference in index (Shannon) of diversity. Abbreviations: MAM, mucosa-associated microbiota; $\mathrm{PBC}$, primary biliary cholangitis; $\mathrm{HCs}$, healthy controls; PERMANOVA, permutational analysis of variance.

\section{a}

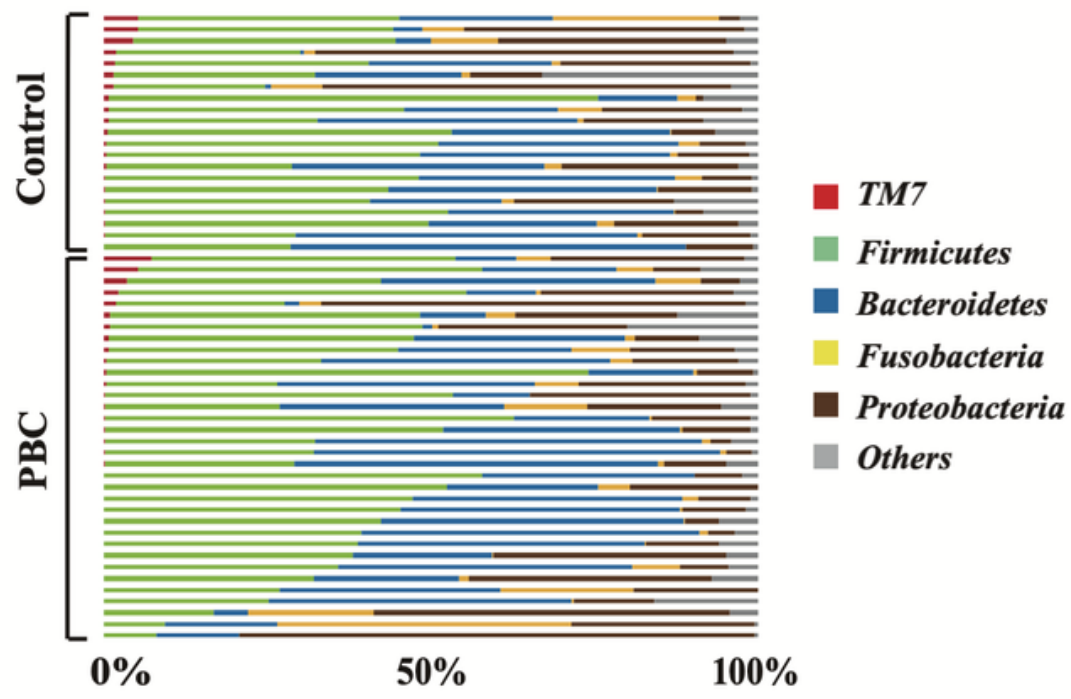

b

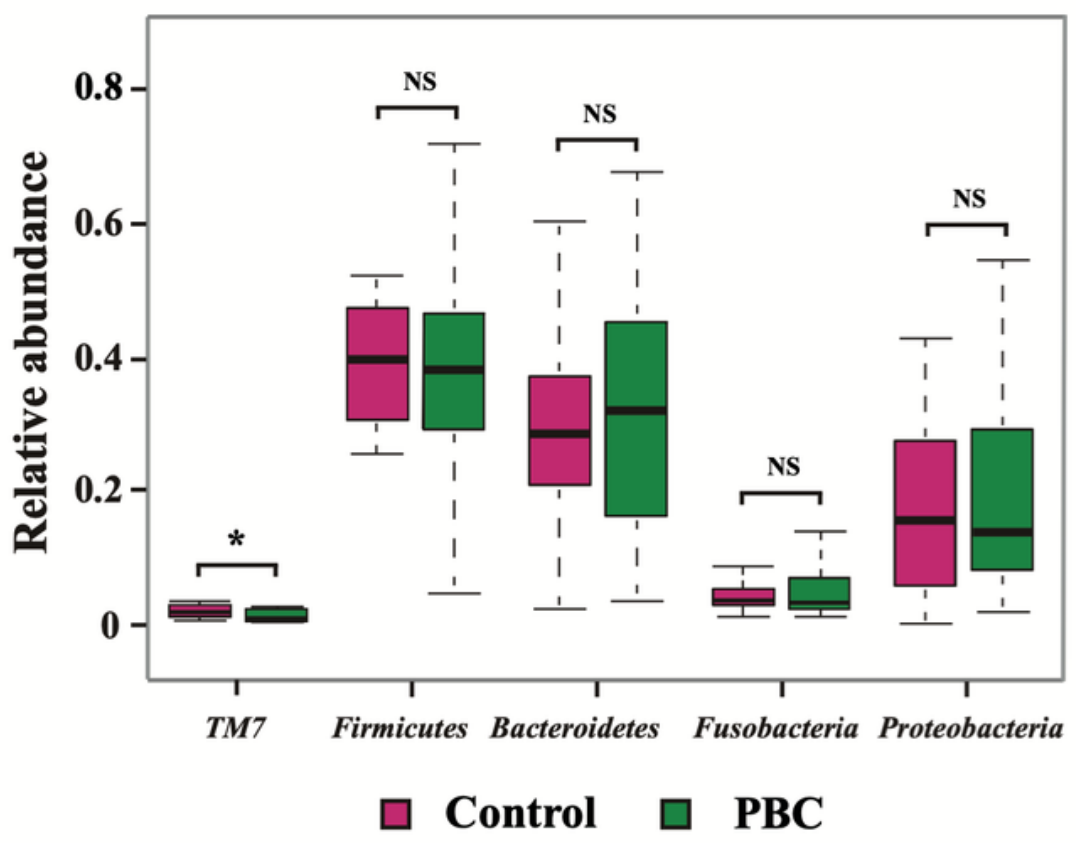

Figure 2

Differences in characterized MAM between patients with PBC and HCs at the phylum level (a) Bacterial composition at phylum level. The relative amount of bacterial composition at the phylum level is shown 
in a bar graph for each sample obtained from the PBC HC groups. (b) Median phylum abundance in the $\mathrm{PBC}$ and $\mathrm{HC}$ groups. Results are expressed as the median \pm standard deviation. The differences were compared using the Wilcoxon rank-sum test. ${ }^{*} \mathrm{P}<0.05$. Abbreviations: MAM, mucosa-associated microbiota; $\mathrm{PBC}$, primary biliary cholangitis; $\mathrm{HCs}$, healthy controls.
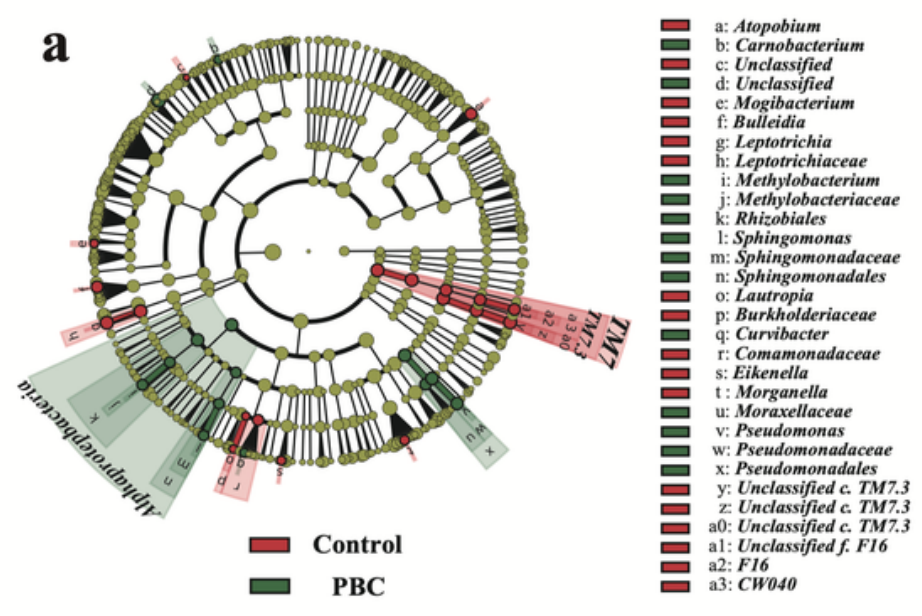

b

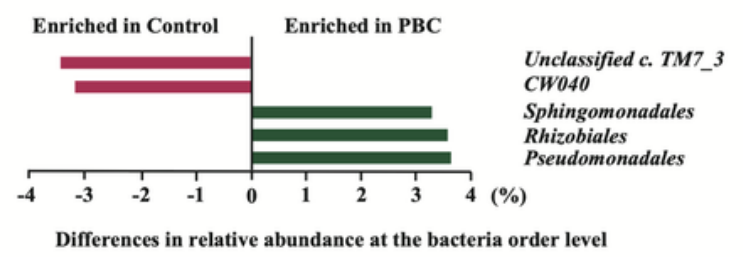

c

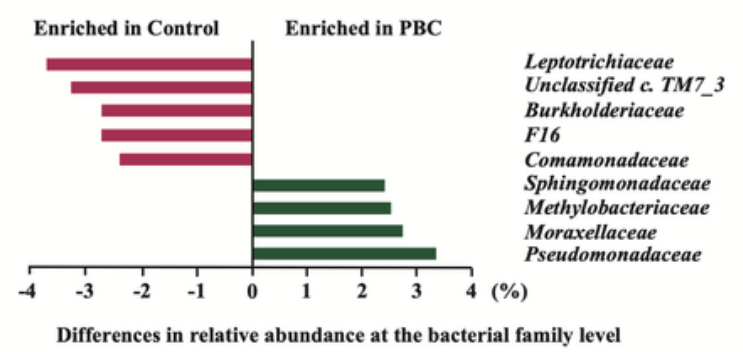

d

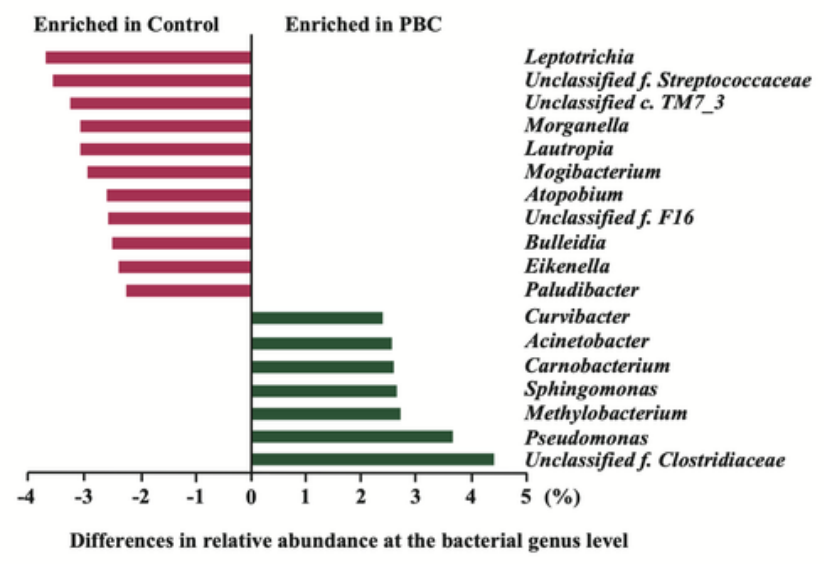

Figure 3 
Changes in gut bacterial taxonomic abundance in patients with PBC $(n=34)$ compared with HCs $(n=21)$ (a) Bacterial taxa identified as rich in differences between the two groups analyzed by LEfSe. Bacterial taxa of the patients with PBC were compared with those of HCs at various levels. Bacterial taxa with high numbers of bacteria in the PBC group are shown in green, and bacterial taxa with high numbers of bacteria in the $\mathrm{HC}$ group are shown in red. In addition, the bacterial taxa showing insignificant differences in their abundance between the two groups are shown in yellow. LEfSe identified Alphaproteobacteria as a differentially abundant taxon in the PBC group versus the HC group. (b) Order-level relative abundance of bacteria in the PBC group compared with that in the HC group. (c) Family-level relative abundance of bacteria in the PBC group compared with that in the HC group. (d) Genus-level relative abundance of bacteria in the PBC group compared with that in the $\mathrm{HC}$ group. Values are expressed as the median of the interquartile range. Abbreviations: $\mathrm{PBC}$, primary biliary cholangitis; $\mathrm{HCs}$, healthy controls; LEfSe, linear discriminant analysis effect size. 


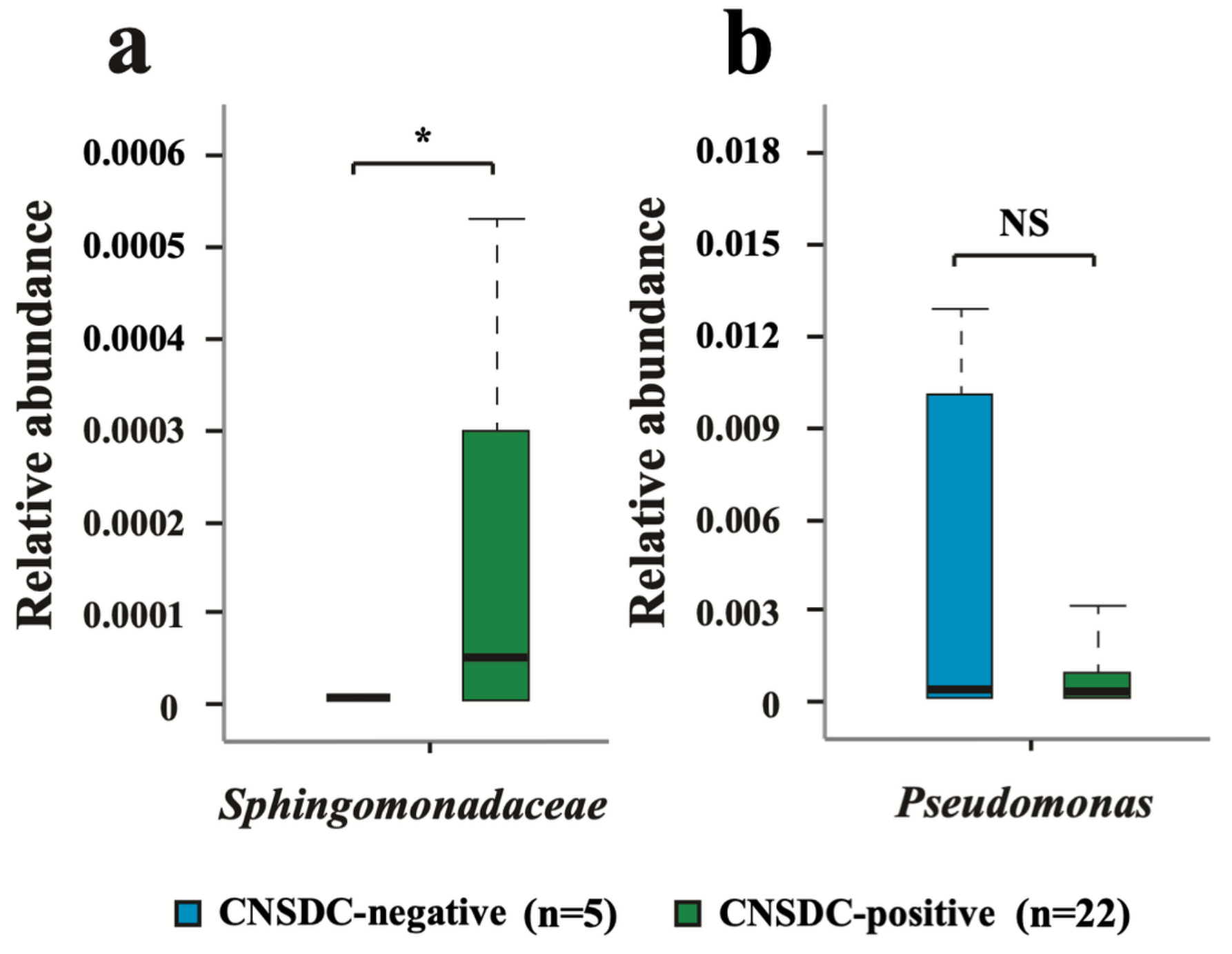

Figure 4

Relative abundance of (a) Sphingomonadaceae and (b) Pseudomonas in the CNSDC-positive PBC group compared with that in the CNSDC-negative PBC group The values are expressed as the median of the interquartile range. ${ }^{*} \mathrm{P}<0.05$ compared with the CNSDC-negative $\mathrm{PBC}$ group. Abbreviations: CNSDC, chronic non-suppurative destructive cholangitis; $\mathrm{PBC}$, primary biliary cholangitis.

\section{Supplementary Files}

This is a list of supplementary files associated with this preprint. Click to download. 
- SupplementaryFigure.1.pdf

- SupplementaryFigure.2.pdf

- SupplementaryFigure.3.pdf

- Supplementarylnformation.docx 